\title{
Rescue and characterization of a local genotype in Sardinia: Apersorgia nera.
}

\author{
Massimino Farci *, Giorgia Marongiu, Dario Campus, Onofrio Graviano, Maria Pia Rigoldi, Alessandra Frau, \\ Gianni Lovicu
}

AGRIS Sardegna, Via Mameli 126/d, Cagliari, Italy;

\begin{abstract}
Apesorgia nera is an ancient Sardinian grape variety present not only in the old vineyards, but also in courtyards and gardens, throughout the island, mainly in Southern and central Sardinia. This work aims to contribute to the ampelographic, chemical and genetic characterization of this autochthonous variety. The comparison of microsatellite profiles obtained with the main "on line" databases did not allow to identify other vines with the same profile. Apesorgia nera is a variety currently being registered in the National Register of Vine Varieties. The long tradition of cultivation, the presence in historical documents, the qualitative and productive characteristics make it an interesting variety, mainly for the local market.
\end{abstract}

Apesorgia Nera è una antica varietà di uva da tavola della Sardegna, diffusa in tutta l'isola e presente non solo nei vecchi vigneti, ma anche in cortili e giardini. Questo lavoro si propone di contribuire alla conoscenza e caratterizzazione ampelografica e genetica del vitigno e nel contempo a contribuire alla conoscenza di una delle varietà più apprezzate e ricercate dai consumatori sardi. Le osservazioni ampelografiche sono state effettuate in quattro anni, dal 2012 al 2015, presso la collezione di germoplasma di vite di Agris Sardegna ad Ussana (CA). Le piante sono innestate su $1103 \mathrm{P}$, allevate a Guyot e hanno un'età di 10 anni. La distanza di impianto è pari a $2,4 \mathrm{~m}$ per $1,0 \mathrm{~m}$, per una densità di impianto di 4166 ceppi per ettaro

\section{Genetica, sinonimie, origine diffusione}

Sinonimie in bibliografia: Appicadorza, Triga, Triga niedda, Trija niedda; Regina nera

Tabella 1. Profilo microsatelliti dell'Apesorgia nera

\begin{tabular}{|c|c|c|c|c|c|c|c|}
\hline \multicolumn{2}{|c|}{ VVS2 } & \multicolumn{2}{c|}{ VVMD5 } & \multicolumn{2}{c|}{ VVMD7 } & \multicolumn{2}{c|}{ VVMD25 } \\
\hline 131 & 135 & 228 & 230 & 240 & 250 & 237 & 253 \\
\hline \multicolumn{2}{|c|}{ VVMD27 } & \multicolumn{2}{|c|}{ VVMD28 } & \multicolumn{2}{c|}{ VVMD32 } & \multicolumn{2}{c|}{ VrZag62 } \\
\hline 183 & 191 & 256 & 258 & 250 & 270 & 186 & 186 \\
\hline \multicolumn{2}{|c|}{ VrZag79 } & \multicolumn{2}{|c|}{ VVIB 01 } & \multicolumn{2}{c|}{ VMC4F 3-1 } & \multicolumn{2}{c|}{ VMC1B 11 } \\
\hline 248 & 254 & 290 & 298 & 186 & 188 & 166 & 188 \\
\hline VVIN 16 & \multicolumn{2}{|c|}{ VVMD 21 } & \multicolumn{2}{c|}{ VVMD 24 } & \multicolumn{2}{c|}{ VVIP 31 } \\
\hline 151 & 151 & 241 & 247 & 206 & 206 & 180 & 190 \\
\hline \multicolumn{2}{|c|}{ VVIV 37 } & \multicolumn{2}{|c|}{ VVIQ 52 } & \multicolumn{2}{c|}{ VVIH 54 } & \multicolumn{2}{c|}{ VVIP 60 } \\
\hline
\end{tabular}

\footnotetext{
Corresponding author: mfarci@agrisricerca.it
}

\begin{tabular}{|c|c|c|c|c|c|c|c|}
\hline 157 & 157 & 81 & 85 & 166 & 168 & 324 & 324 \\
\hline & & \multicolumn{2}{|c|}{ VVIV 67} & \multicolumn{2}{|c|}{ VVIN 73} & & \\
\hline & & 358 & 358 & 263 & 263 & & \\
\hline
\end{tabular}

Corrispondenze sarde accertate per profilo genetico: Aghina de Gerusalemme di Abbasanta, Litachina e Trija Niedda di Mores.

Corrispondenze italiane ed estere accertate per profilo genetico: nessuna

False attribuzioni: si ritrova in bibliografia la citazione di "Apesorgia niedda" come sinonimo del vitigno francese Prune de Cazoul, che pur essendo diverso condivide 26 alleli con la nostra Apesorgia. Uno degli indici di vicinanza genetica è dato dal numero di alleli condivisi che si accompagna all'analisi filogenetica con il dendrogramma che, oltre al numero di alleli condivisi e della loro dislocazione nei diversi loci, tiene conto anche della frequenza nella popolazione degli alleli in comune.

Tabella 2. Elenco dei vitigni che condividono almeno il 50\% degli alleli con Apesorgia nera.

\begin{tabular}{|c|c|}
\hline Denominazione & $\begin{array}{c}\mathbf{n}^{\circ} \text { alleli condivisi } \\
\text { Su 44 }\end{array}$ \\
\hline Apesorgia bianca & 28 \\
\hline Saluda e passa & 27 \\
\hline Retagliadu & 25 \\
\hline Monica Bianca & 24 \\
\hline Albaranzeuli Nero & 23 \\
\hline Zirone Bianco & 23 \\
\hline Albaranzeuli Bianco & 22 \\
\hline Mara bianca & 22 \\
\hline Cannonau rosato & 22 \\
\hline Fiudedda & 22 \\
\hline Isabella falsa & 22 \\
\hline Niedda Manna & 22 \\
\hline
\end{tabular}

Fig. 1. Particolare del dendrogramma 


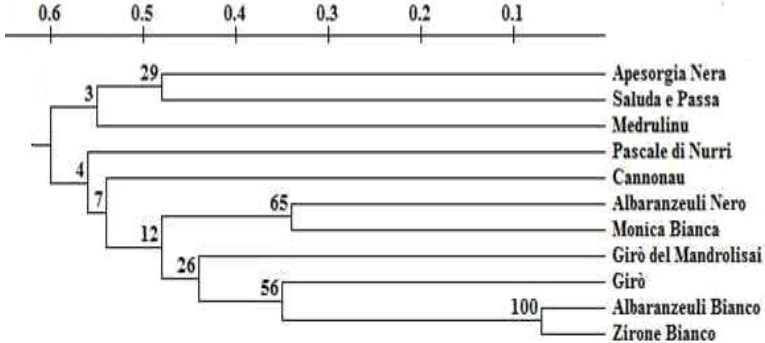

L'Apesorgia nera 2 presenta una piccola differenza nel profilo SSR: al locus VrZag79 è omozigote (248 - 248).

L'Apesorgia nera sembrerebbe aver dato origine all'Apesorgia bianca in seguito ad incrocio con l'Axina de tres bias.

Tabella 3. Elenco dei vitigni che condividono almeno un allele per locus con Apesorgia nera

\begin{tabular}{|c|c|}
\hline Vitigno & $\begin{array}{c}\text { Rapporto } \\
\text { parentela }\end{array}$ \\
\hline Apesorgia bianca & figlio \\
\hline Saluda e passa & nd \\
\hline Retagliadu & nd \\
\hline
\end{tabular}

Essa risulta anche compatibile per un rapporto di parentela diretta con la varietà Saluda e passa e con il Retagliadu. Il dendrogramma mostra una stretta vicinanza con la Saluda e passa, con la quale condivide 27 alleli. Come la bianca, anche l'Apesorgia nera sembra essere al momento un vitigno autoctono per il quale non è stata ritrovata nessuna corrispondenza se non nei vitigni locali. E' un'uva da tavola tradizionale, coltivata in tutta l'isola e ancora molto apprezzata. E' la Trigia longa ricordata dal Manca dell'Arca e qualche anno più tardi il Moris cita la varietà 'jucunda', in lingua locale denominata "Apesorgia niedda". L'Angius la individua nel Campidano di Cagliari, Marmilla, Trexenta e Ogliastra. Il Cettolini, 50 anni piu' tardi, ne accerta la presenza nelle altre aree dell'isola. A questa varietà è riconducibile una delle viti monumentali più antiche individuate nell'isola, in agro di Mandas. Anche per questo vitigno non si conoscono varietà affini di vite esterne all'isola. Anche la Apesorgia nera è pertanto allo stato delle cose da considerarsi un "unicum". Zone di coltivazione in Sardegna. Tutta l'isola.

\section{Ampelografia}

\subsection{Germoglio giovane}

Fig. 2. Il germoglio giovane di Apesorgia nera

Apice del germoglio: completamente aperto, con distribuzione della pigmentazione antocianica dei peli striscianti assente e di intensità nulla o molto bassa. La densità dei peli striscianti è nulla o molto bassa. La densità dei peli eretti è nulla o molto bassa.

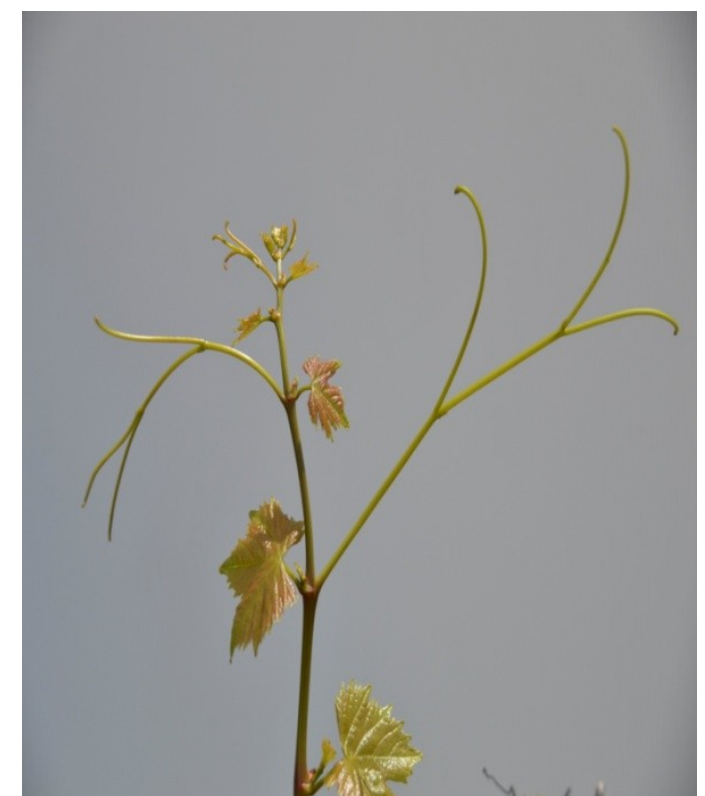

Fig. 2. Il germoglio giovane di Apesorgia nera

\subsection{Foglia giovane}

Il colore della pagina superiore del lembo della foglia giovane è rosso-ramato. La densità dei peli striscianti della pagina inferiore della foglia è nulla o molto bassa. La densità dei peli eretti è nulla o molto bassa.

\subsection{Germoglio e viticci}

Il germoglio si presenta con un portamento semieretto o orizzontale, con il colore del lato dorsale degli internodi e dei nodi verde e rosso e il colore del lato ventrale di internodi e nodi verde. Gli internodi presentano la densità dei peli eretti nulla o molto bassa, e la densità dei peli striscianti nulla o molto bassa.

Viticci: i viticci consecutivi in numero non superiore a due, si presentano molto lunghi, talvolta lunghi, con una lunghezza di 25 e anche 30 centimetri.

\subsection{Fiore}

Il fiore è ermafrodita autofertile con stami e gineceo completamente sviluppati. La prima infiorescenza è generalmente inserita dopo il $5^{\circ}$ nodo. Nel corso delle osservazioni, è stato osservato un numero medio di infiorescenze per germoglio minore di 1 , in individui performanti può anche superare un infiorescenza per germoglio.

\subsection{Foglia adulta}

La foglia adulta è media o grande, di forma cuneiforme, eptilobata. Il colore è verde medio, con pigmentazione antocianica sulle nervature principali della pagina superiore del lembo assente. Il profilo della sezione trasversale della foglia è involuto. La bollosità della pagina superiore del lembo è bassa o molto bassa. I denti della foglia adulta si presentano con entrambi i lati rettilinei. La dimensione dei denti in rapporto alla lamina 
fogliare è grande, con i denti che sono mediamente lunghi quanto larghi. Il picciolo si presenta più corto della nervatura principale della foglia. Il seno peziolare ha una forma a $\mathrm{V}$ ed è aperto. Nel seno peziolare non si nota la presenza di denti e la base del seno della foglia non è delimitata su entrambi i lati dalla nervatura. I margini dei seni laterali superiori, generalmente poco, talvolta mediamente, profondi, si presentano leggermente sovrapposti. La densità dei peli striscianti e la densità dei peli eretti della pagina inferiore della foglia è nulla o molto bassa. La densità dei peli striscianti sulle nervature della pagina inferiore della foglia è nulla o molto bassa e quella dei peli eretti sulle stesse nervature è nulla o molto bassa.

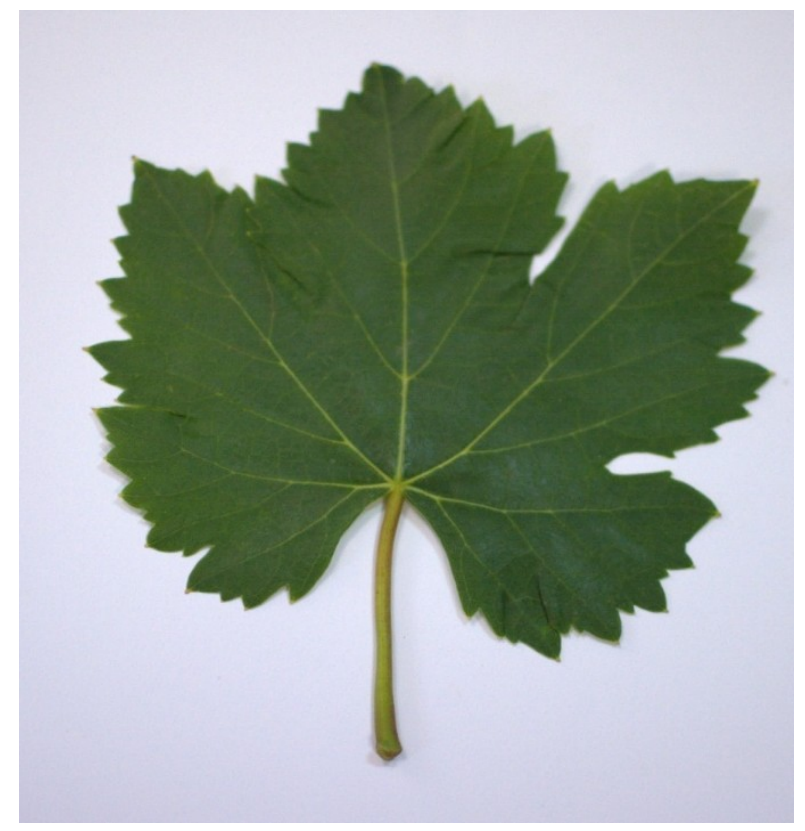

Fig. 3. Foglia adulta di Apesorgia nera

Lunghezza della foglia: $\mathrm{mm} \mathrm{163,5}( \pm 14,0)$.

Larghezza della foglia: $\mathrm{mm} \mathrm{153,2}( \pm 12,4)$.

Lunghezza del picciolo: $\mathrm{mm} 75,9( \pm 17,6)$.

Lunghezza della nervatura N1, N2, N3, N4, N5: mm $118,0( \pm 8,6), \mathrm{mm} \mathrm{110,6}( \pm 9,5), \mathrm{mm} \mathrm{76,4}( \pm 7,2), \mathrm{mm}$ $50,3( \pm 6,5), \mathrm{mm} 24,0( \pm 4,0)$.

Angolo tra N1 e N2 misurato alla prima biforcazione: $52,9^{\circ}( \pm 0,1)$.

Angolo tra $\mathrm{N} 2$ e N3 misurato alla prima biforcazione: $56,0^{\circ}( \pm 1,5)$.

Angolo tra N3 3 N4 misurato alla prima biforcazione: $56,8^{\circ}( \pm 1,6)$.

Rapporto tra la lunghezza del picciolo e la lunghezza della nervatura N1: 0,6 .

Stima dell'area della fogliare: $\mathrm{mm} 2 \quad 17673,3( \pm 3339,4)$.

\subsection{Tralcio legnoso}

Il tralcio legnoso, di colore prevalente brunastro, presenta una sezione trasversale circolare, con una struttura della superficie striata. I peli eretti sono assenti sui nodi e sugli internodi. Il diametro degli internodi intorno agli 8 millimetri, ha dimensioni piccole.

\subsection{Grappolo, acino e i dati dell'uva}

Il grappolo, di taglia bassa pesa 275,0 grammi $( \pm 25,0)$, presenta una lunghezza media di 230,0 millimetri $( \pm$ $27,0)$ e una larghezza media di 127,1 millimetri $( \pm 14)$, che lo definiscono rispettivamente come molto lungo e mediamente largo.

Il peduncolo del grappolo principale presenta una lunghezza media di 61,2 millimetri $( \pm 11,0)$ e risulta pertanto mediamente lungo.

Il grappolo di Apesorgia nera è molto spargolo o spargolo, con una forma ad imbuto e con 1-2 ali presenti.

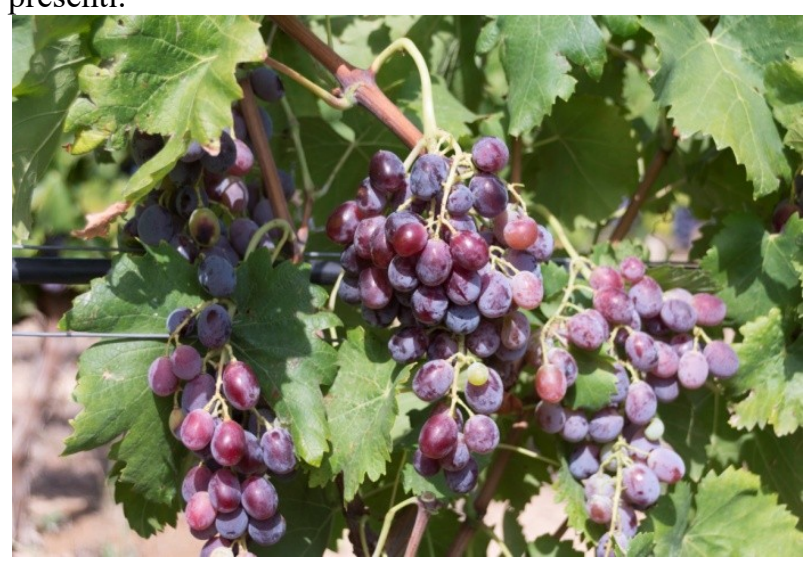

Fig. 4. Grappolo di Apesorgia nera

L'acino è di peso medio (grammi 4,7 $\pm 0,1$ ), lungo ( $\mathrm{mm}$ $24,0 \pm 1,0)$; mediamente largo (mm 19,7士 1,0); ellissoidale largo, con sviluppo dei vinaccioli completo. La buccia si presenta di colore rosso scuro violetto; con una pigmentazione antocianica della polpa nulla o molto debole, poco succosa, molto soda. L'acino non presenta sapore particolaree si distacca difficilmente dal pedicello.

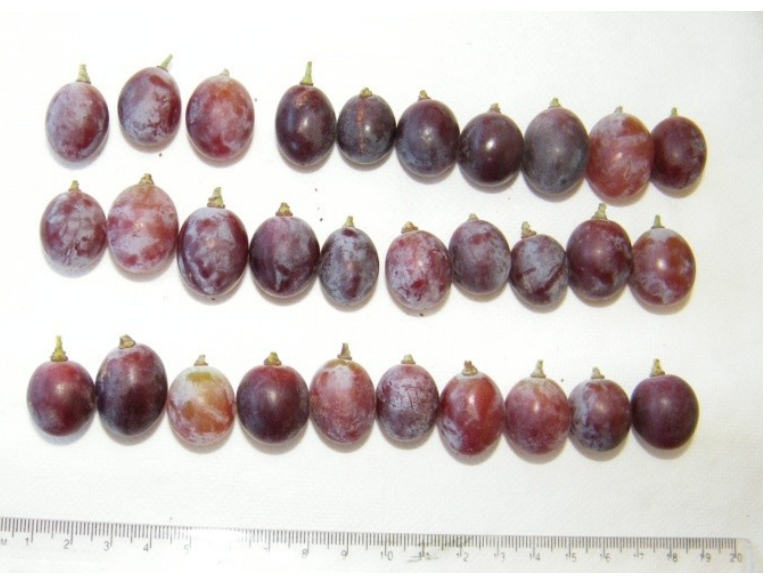

Fig. 5. Acini di Apesorgia nera

Utilizzazione: tradizionalmente come uva da tavola. Tenore in zucchero del mosto: $19,2^{\circ} \mathrm{Brix}( \pm 3,4)$ Acidità totale del mosto: $4,0 \mathrm{~g} / \mathrm{l}( \pm 0,9)$.

$\mathrm{pH}$ del mosto: $3,7( \pm 0,2)$

Acido malico: $1,0 \mathrm{~g} / 1( \pm 0,4)$.

Acido tartarico: $5,5 \mathrm{~g} / 1( \pm 1,2)$. 
Polifenoli totali: $440,6 \mathrm{mg} / \mathrm{l}( \pm 64,4)$.

Flavonoidi totali (in percentuale rispetto a Cannonau): $156,8 \%$.

Antociani totali (in percentuale rispetto a Cannonau): $82,6 \%$.

\subsection{Fenologia}

Germogliamento: prima decade di aprile, come Cannonau.

Fioritura: terza decade di maggio, come Cannonau.

Invaiatura: seconda decade di agosto, circa 2 settimane dopo Cannonau.

Maturazione dell'uva: terza decade di settembre, una settimana prima di Cannonau.

Fertilità potenziale per lo sperone: $0,80( \pm 0,3)$.

Fertilità potenziale per il capo a frutto: 1,20 ( $\pm 0,3)$.

Resistenza alle malattie: mediamente sensibile alla peronospora.

Produzione. Nel corso dei tre anni, la produzione media riscontrata tra le diverse accessioni, è stata di 2,3 $\mathrm{Kg} /$ ceppo $( \pm 0,8)$.

\section{Conclusioni}

L'Apesorgia nera è una varietà a buccia rossa non presente nel Registro nazionale delle Varietà di Vite. Il confronto dei profili microsatelliti ottenuti con le principali Banche Dati "on line" non ha permesso di individuare altri vitigni con lo stesso profilo.

L'Apesorgia nera è una varietà in corso di iscrizione nel Registro nazionale delle Varietà di Vite. L' "unicum" accertato dalla comparazione con circa 2500 profili SSR, la lunga tradizione di coltivazione, la presenza in documenti storici, le caratteristiche qualitative e produttive la rendono una varietà interessante, principalmente sia per il consumatore locale che per il consumatore alla ricerca di prodotti dal gusto e dall'aspetto differente.

\section{Bibliografia}

1. V. Angius. Città e villaggi della Sardegna dell'Ottocento (3 volumi). Edizioni Ilisso. Nuoro. (2006)

2. AA.VV., AKINAS Uve di Sardegna. Edizioni Ilisso. Nuoro. 2017. ISBN-13: 978-8862023528

3. AA.VV.. La coltivazione dell'uva da tavola in ambiente mediterraneo. ISBN 978-8890340406. (2008)

4. A. Cara. Schizzo di Vocabolarietto ampelologico comprendente le varietà di vite coltivate in Sardegna. Stab. tip. Serreli, Cagliari. (1909)

5. S. Cettolini. Regia Scuola di Viticoltura ed Enologia di Cagliari. Annuario per gli anni scolastici, 3,1893-1894.

6. G. Lovicu, M. Farci, M. Sedda, M. Labra, F. De Mattia, F. Grassi, G. Bacchetta, M. Orrù. L'Informatore Agrario, 34, 40-41. (2010)
7. A. Manca dell'Arca. Agricoltura di Sardegna. Edizioni Ilisso. Nuoro. (2000)

8. Moris J., 1837. Flora Sardoa, Torino.

9. O.I.V.. Lista dei caratteri descrittivi OIV delle varietà e specie di Vitis (2a edizione). (2009).

10. Online resource 4 (Lacombe 2012) https://static-content.springer.com/esm / art\%3 A10. 1007\% 2Fs00122-012-1988-2/MediaObjects/ 122_2012_1988_MOESM4_ESM.pdf

11. Online resource 5 (Lacombe 2012) https://static-content.springer.com/esm/ art\%3A10.1007\%2Fs00122-012-19882/MediaObjects/122_2012_1988_MOESM5_ESM. pdf

12. Registro nazionale delle varietà della vite. http://catalogoviti.politicheagricole.it/catalogo.php 